\title{
COMBINED STERILE INSECT TECHNIQUE AND INCOMPATIBLE INSECT TECHNIQUE: CONCEPT, STUDY DESIGN, EXPERIENCE AND LESSONS LEARNED FROM A PILOT SUPPRESSION TRIAL IN THAILAND
}

\author{
P. KITTAYAPONG ${ }^{1,2}$ \\ ${ }^{I}$ Center of Excellence for Vectors and Vector-Borne Diseases, Faculty of Science, \\ Mahidol University, 999 Phuttamonthon 4 Road, Nakhon Pathom 73170, Thailand \\ ${ }^{2}$ Department of Biology, Faculty of Science, Mahidol University, 272 Rama VI \\ Road, Bangkok 10400, Thailand; pkittayapong@gmail.com
}

\begin{abstract}
SUMMARY
Climate change, rapid global transport and land use change leading to urbanization and agricultural intensification have facilitated disease emergence in vulnerable regions like Southeast Asia, and also the global expansion of vectors and vector-borne diseases into other regions like the Americas and Europe. Important vector-borne diseases, i.e. dengue, chikungunya, yellow fever, and Zika are transmitted by the major mosquito vector species, Aedes aegypti (L.) and Aedes albopictus (Skuse). Management of Ae. aegypti populations in countries endemic to these diseases, especially in Southeast Asia, is not sufficiently effective, resulting in high morbidity and mortality in the region. Insecticide resistance has become an important issue, causing failure in insecticide-based vector control. Innovative or alternative tools/approaches are needed to effectively reduce mosquito vector populations and consequently reduce the diseases they transmit. A trial integrating the environment-friendly Sterile Insect Technique (SIT) and the insect incompatible technique (IIT) was successfully carried out on a small-scale in a semi-rural setting in Thailand. In this chapter, we report on the design and methodology, as well as the experience and lessons learned from the baseline preparation and implementation of the pilot trial.
\end{abstract}

Key Words: Aedes aegypti, Aedes albopictus, SIT, IIT, Wolbachia, mosquito control, integrated vector management, vector-borne diseases

J. Hendrichs, R. Pereira and M. J. B. Vreysen (eds.), Area-Wide Integrated Pest Management: Development and Field Application, pp. 405-432. CRC Press, Boca Raton, Florida, USA.

(C) 2021 IAEA 


\section{INTRODUCTION}

Vector-borne diseases are becoming increasingly a public health problem and globally a significant economic burden. According to the World Health Organization (WHO), about half of the world's people in over 100 countries are at risk of contracting dengue (WHO 2019a). Chikungunya, another viral disease transmitted to humans by mosquito vectors, was originally confined to Africa but has recently been spreading rapidly across the Indian Ocean, Europe, the Americas, Asia, and Oceania. In the last decade, outbreaks of Zika in several parts of the world epitomized the need for new and effective methodologies to manage mosquito populations vectoring these diseases.

With the number of dengue cases and the number of countries affected rising dramatically in recent years, the socio-economic impact of mosquito-transmitted diseases is enormous. The overall estimated annual economic burden of dengue in Southeast Asia was USD 950 million, with the average annual direct costs being USD 451 million and the indirect costs being USD 499 million (Shepard et al. 2013). In Thailand alone, a recent study estimated the mean economic cost of dengue at USD 135 million per annum (Shepard et al. 2013). In the absence of affordable and effective vaccines and drugs to combat dengue, chikungunya, and Zika, population control of mosquito vectors is the most effective way of managing these diseases. Most vector control strategies are insecticide-based, and their widespread use has resulted in increased insecticide resistance among the mosquitoes. Therefore, there is an urgent need for alternative novel approaches for vector control.

Aedes aegypti (L.), the yellow fever mosquito, is considered the main mosquito vector for dengue, chikungunya, and Zika in many parts of the world (Calvez et al. 2017; Kotsakiozi et al. 2017; Trewin et al. 2017). Attempts have been made to control this invasive species, but traditional mosquito control methods, such as insecticide applications and source reduction by eliminating larval breeding sites have been insufficient for suppressing this mosquito vector and reducing disease incidences (Fredericks and Fernandez-Sesma 2014; Trewin et al. 2017).

Several novel Ae. aegypti control methods, namely the Sterile Insect Technique (SIT) that is based on the release of irradiated sterile males (Dyck et al. 2021); the Incompatible Insect Technique (IIT), which depends on Wolbachia-induced cytoplasmic incompatibility (CI) by releasing Wolbachia-infected males (Bourtzis et al. 2014, Mains et al. 2016; Zheng et al. 2019); and the application of genetically modified mosquito strains, such as those carrying RIDL (Release of Insects carrying a Dominant Lethal) constructs (Thomas et al. 2000; Morrison et al. 2010; Carvalho et al. 2015), have recently been endorsed by the WHO to help contain the recent Zika virus outbreak (Zheng et al. 2015; Yakob et al. 2017; WHO 2019b).

Both the SIT and the IIT are based on the repeated inundated release of large numbers of high quality sterile male mosquitoes to compete with their wild male counterparts in mating with wild females in a target area, thus inducing female sterility, which results in a reduction in the target populations (Zheng et al. 2015; Mains et al. 2016; WHO 2017; Zhang et al. 2017; Zheng et al. 2019) and consequently a potential reduction or prevention of the transmission of mosquito-borne diseases.

As a component of area-wide integrated pest management (AW-IPM) programmes, the implementation of the SIT and the IIT depends on several important 
components, including mass-rearing, sex separation, sterilisation, transportation, release, and monitoring (Zhang et al. 2017; Nikolouli et al. 2018; Dyck et al. 2021). Hence, the number of released sterile males must significantly surpass the number of wild males in the release area to compensate for any negative effect associated with domestication, mass-rearing, storage, and their overall handling, so that they can compete with wild males for matings with wild females, allowing the introduction of sufficient sterility into the wild populations (Vreysen et al. 2007; Barnes et al. 2015; Nikolouli et al. 2018; Dyck et al. 2021).

The combination of Wolbachia-induced IIT and the SIT was applied together with initial source reduction to suppress natural populations of Ae. aegypti in a semi-rural village in Chachoengsao Province, eastern Thailand. Results of this pilot trial indicated successful reduction of local Ae. aegypti populations after 6 months of repeated releases of sterile males (Kittayapong et al. 2019).

In this chapter, we report on the study design and methodology of this pilot trial. In addition, experience and lessons learned from the baseline experiments and from the implementation of this small-scale pilot trial are discussed.

\section{COMBINED SIT/IIT APPROACH: CONCEPT AND PROGRESS}

\subsection{Sterile Insect Technique (SIT) for Mosquito Control}

The SIT is a method of insect pest control with a strong record of success against a wide range of agricultural pests and which potentially can work against mosquitoes (Dyck et al. 2021). The technique consists of repeated area-wide releases of large numbers of sterile males in the target area, where they will mate with native females. Eggs will be produced but they will not hatch. When adequate sterile to wild male overflooding ratios are maintained, the number of native insects decreases with each generation, potentially driving the native population to very low numbers or, under complete isolation, to local extinction.

The SIT has been successfully implemented in large-scale operations to control agricultural insect pests and to prevent losses in livestock or crops of economic importance. Because it has no environmental impact and its relatively unobtrusive means of deployment, the SIT had been well accepted, even in urban areas. This technique has been successfully proven for over 50 years and is cost-effective for the population control of some major agricultural and livestock pests (Vreysen et al. 2000; Dyck et al. 2021). For public health pests, the SIT has been the subject of extended research since the late-1950s. However, it has never reached an operational level (Dame et al. 2009), even though it is considered to be a highly sustainable and environment-friendly method with, so far, no negative effect on human health (Alphey et al. 2010).

The first experimental sterile mosquito releases were conducted by the United States Department of Agriculture (USDA) in southern Florida. A total of 32000 sterile Anopheles quadrimaculatus Say males that emerged from pupae irradiated with 120 Gy were released for three months in 1959 and in 1960; this amount was increased to 300000 released over a period of nine months (Weidhaas et al. 1962; 
Dame et al. 1964, 2009). However, the project was considered not successful as insufficient sterility was induced in the wild population (Dame et al. 1964, 2009).

The Centers for Disease Control and Prevention (CDC) carried out a release trial in Pensacola, Florida with 110-180 Gy treated Ae. aegypti. Although 3.9 million sterile males were released over four months in 1960 and 6.7 million over six months in 1961, the project was considered a failure due to reduced sterile male competitiveness caused by the irradiation of the pupae (Morlan et al. 1962; Dame et al. 2009).

Between 1967 and 1974, the World Health Organization/Indian Council of Medical Research (WHO/ICMR) and the USDA released male Culex quinquefasciatus Say irradiated with 60-120 Gy in India and Florida, respectively. The daily release rate ranged between 9000 to 15000 sterile males. Nevertheless, these studies confirmed previous laboratory findings that the somatic damage was greater when younger pupae were treated as compared with older pupae (Patterson et al. 1975, 1977; Dame et al. 2009).

In 1980, a total of 71000 sterile Culex tarsalis Coquillett males, sterilized by 60 Gy irradiation at the adult stage, were released in California, USA and results showed that these sterile males were fully competitive. However, in 1981, 85000 sterile males were released, but these sterile males were not capable of seeking out the wild females and transferring the sterile sperm (Reisen et al. 1982; Dame et al. 2009).

Mosquito releases have been carried out for numerous purposes related to SIT application, but most of them were directed at answering a specific research question without any anticipation of population suppression. However, a few suppression and/or elimination projects have been attempted, but only modest effects were observed on sterility of the oviposited eggs and reduction of the wild population density (Benedict and Robinson 2003).

More recently, several SIT pilot projects have been initiated to answer specific questions (Lees et al. 2021). The effect of irradiation on sexual maturation and mating success of males, and the sexual competitiveness of sterile versus wild males in the presence of wild females of Aedes albopictus (Skuse) were studied under semi-field conditions in La Réunion Island (Oliva et al. 2012). In Sudan, participation of irradiated Anopheles arabiensis Patton males in mating swarms during the evening after their release was demonstrated, but their competitiveness and achieving successful copulation in the field was not proven (Ageep et al. 2014).

In Mauritius, the Ministry of Health and Quality of Life has been developing an operational plan to assess the SIT for population reduction of Ae. albopictus to prevent and control chikungunya and dengue, and guidelines for site selection were developed with the beginning of population surveillance (Iyaloo et al. 2014).

The first successful SIT mosquito pilot project was initiated during the summer of 2004 in three small towns in northern Italy. Approximately 900-1600 irradiated Ae. albopictus pupae were released per hectare, per week, and this continued for five years. The trial induced up to $68 \%$ egg sterility in the target population, demonstrating the potential of sterile males to suppress populations of Ae. albopictus (Bellini et al. 2007, 2013; Lees et al. 2015). 
To date, there have been no large operational mosquito SIT projects, but operational programmes should eventually become established and more efficient over time (Dame et al. 2009). Experimentation and preparation processes for SIT application tend to be longer-term. Also, it does not have immediate effects on vector numbers, but impacts the size of the wild population in the next generation. In addition, entomological surveillance of the vector population before and during releases is essential to monitor the impact of any releases (Dame et al. 2009; Alphey et al. 2010). Nevertheless, the SIT is robust in term of both efficacy and cost when used in combination with other compatible methods, resulting in successful and sustainable vector control. Apart from being an environmentally-sound biological control approach, the SIT can be easily integrated with other biological control strategies (parasitoids, predators, and pathogens) (Vreysen et al. 2007; Barnes et al. 2015; Nikolouli et al. 2018).

\subsection{Wolbachia-based Approach for Mosquito Control}

Wolbachia are intracellular endosymbionts belonging to Alpha-proteobacteria. They are found in many arthropods and nematodes, and the overall species infection rate is as high as $66 \%$ (Hilgenboecker et al. 2008). Wolbachia bacteria have attracted the interest of the scientific community because of their potential to block arbovirus infections in mosquitoes (Moreira et al. 2009; Bian et al. 2013), as well as their capacity to replace natural populations of insects through their CI properties (Turelli and Hoffmann 1995). Wolbachia-infected male insects are not compatible with their non-infected natural females, leading to a reduction in the egg hatch of Wolbachiauninfected populations and then the replacement by Wolbachia-infected populations (Hoffmann et al. 2011). The benefit of CI has been widely recognized for mosquito vector control (Clark et al. 2002; Atyame et al. 2014; Altinli et al. 2018; Baton et al., this volume).

A few years after the development of Wolbachia-transinfected Aedes mosquitoes (Xi et al. 2005), open field releases of these mosquitoes were carried out to evaluate whether CI induced by Wolbachia and their antiviral ability could be used for population suppression in vector control programmes. An open field release of Aedes polynesiensis Marks fluorescent-marked males infected with Wolbachia was launched in French Polynesia in 2009. The study showed that Wolbachiatransinfected Ae. polynesiensis males were competitive under field conditions; and after 30 weeks of releases, the egg hatch rate was significantly reduced in the release area, resulting in a reduction of the density of the local mosquito population (O’Connor et al. 2012).

The feasibility of using Wolbachia triple infected Ae. albopictus as a biopesticide against natural Wolbachia double infected Ae. albopictus was demonstrated in Lexington, USA and Guangzhou, China. Both the egg hatch rate and the number of adult Ae. albopictus were significantly reduced following the release of Wolbachiainfected males in these trials (Mains et al. 2016; Zheng et al. 2019). In addition, IIT was demonstrated to successfully suppress natural populations of Ae. aegypti in South Miami, USA in order to prevent the Zika disease by releasing wAlbB Wolbachiainfected Ae. aegypti males (Mains et al. 2019). 
Strict male release is required for IIT application to obtain vector suppression (O'Connor et al. 2012; Nikolouli et al. 2018; Baton et al., this volume). Indeed, the accidental release of females infected by Wolbachia may cause the replacement of the targeted population by a population carrying the Wolbachia infection, resulting in field populations being compatible with the released males. Therefore, IIT application requires the development of an efficient method for sex separation at mass-rearing scales, in order to strictly release only Wolbachia-infected males (O’Connor et al. 2012; Nikolouli et al. 2018). Different techniques like phenotypic sorting or genetic sexing methods based on classical genetic or molecular methods have been reported for separation or sexing methods (Gilles et al. 2014). However, these methods are not available for all target species, and some techniques involve the release of genetically modified organisms (GMOs), the use of which is of concern in the European Union, as they face public opposition. In addition to public acceptance, GMO releases also face regulation difficulties in some countries, including China and India (O’Connor et al. 2012; Nikolouli et al. 2018).

In Australia, a risk analysis was carried out before the first release of Wolbachiatransinfected Ae. aegypti male and female mosquitoes into the environment for the purpose of population replacement (Murray et al. 2016). The first release into the field of Ae. aegypti males and females infected with $w \mathrm{Mel}$ was approved and took place in 2011 near Cairns in north-eastern Australia. The study showed that Wolbachiatransinfected Ae. aegypti successfully invaded and completely replaced uninfected wild Ae. aegypti populations (Hoffmann et al. 2011). A follow-up study indicated that field $w$ Mel-infected Ae. aegypti mosquitoes $\left(\mathrm{F}_{1}\right)$, collected one year following the field release, had very low levels of dengue virus replication and dissemination. The frequency of $w$ Mel-infected Ae. aegypti remained at more than $90 \%$ in the mosquito populations for more than 3 years (Frentiu et al. 2014). The success of this first release has led to small- and large-scale releases of wMel-transinfected Ae. aegypti in other countries, in order to evaluate the effectiveness of population replacement in controlling dengue disease in human populations (Joubert et al. 2016).

\subsection{Development of the Combined SIT/IIT-based Approach}

Much progress has been made in recent years towards developing the required technology and methodology to bring mosquito sterility to field application. Hence, pilot releases have begun in a number of sites around the world (Lees et al. 2015). Since the key mosquito disease vectors are all relatively amenable to colonization and rearing, and in many situations the natural population densities are low, the SIT, the IIT, or a combination of the two, are well suited for their management (Lees et al. 2015). The SIT/IIT combination could in principle be applied to any targeted species for which an adequate and highly effective sexing system is not available (Zhang et al. 2015a, 2015b, 2016; Nikolouli et al. 2018; Zheng et al. 2019). However, successful SIT/IIT programmes will also depend on having Wolbachia strains with good CI and maternal transmission phenotypes, apart from an effective sexing system. 
As the female mosquitoes are more radiation sensitive than males, the minimum dose of radiation that leads to complete sterility in females, whilst not negatively affecting male mating competitiveness, has been identified (Zhang et al. 2015b, 2016, 2017; Nikolouli et al. 2018). As a result, any accidentally released Wolbachia-infected females are sterile, and the risk of population replacement is minimised (Lees et al. 2015; Bourtzis et al. 2014; Zhang et al. 2017; Nikolouli et al. 2018; Dyck et al. 2021). Integration of the low irradiation dose with CI when using the virus-resistant strains of Wolbachia also minimizes any potential disease transmission by accidentally released sterile females. This has proven to be an efficient strategy in programmes targeting population suppression of Ae. albopictus (Zhang et al. 2015a, 2015b, 2016; Nikolouli et al. 2018; Zheng et al. 2019) and Ae. aegypti (NEA 2019; Liew et al., this volume). As stated by the WHO, this combined SIT/IIT technology has potential for long-term control of Ae. aegypti and Ae. albopictus mosquito populations, and this approach is considered an effective and safe strategy for the management of mosquito populations (WHO 2017).

\subsection{Combined SIT/IIT Pilot Trial in Thailand}

A field application of the combined SIT/IIT approach to reduce a local Ae. aegypti population was first demonstrated on a small-scale pilot trial with a total study area of $2.19 \mathrm{~km}^{2}$ in the Plaeng Yao District of Chachoengsao Province, in eastern Thailand. Using the direct microinjection method, two Wolbachia strains from Ae. albopictus collected from rubber plantations in Thailand were introduced into Ae. aegypti. This newly developed Ae. aegypti line produced progeny infected with the Wolbachia strains $w \mathrm{AlbA}$ and $w \mathrm{AlbB}$, with maternal transmission efficiency as high as $85 \%$ after 6 generations (Ruang-areerate and Kittayapong 2006). For the combined SIT/IIT approach, the CI property of Wolbachia was used to sterilize natural Ae aegypti mosquito vector populations, while radiation was used to avoid population replacement by assuring that no fertile females were accidentally released.

Once released into nature, Wolbachia-transinfected Ae. aegypti male mosquitoes not only induced sterility in the females of the natural populations, but any potential virus transmission was also blocked in case a few female mosquitoes were inadvertently present in the releases into nature (Moreira et al. 2009). As the SIT/IT method aims at developing a vector suppression tool that is environment-friendly, no propagation of released mosquitoes should happen in nature. To achieve this goal, sterility of the Wolbachia-transinfected male mosquitoes was ensured by exposing the males to an appropriate irradiation dose (Kittayapong et al. 2018).

\section{STUDY DESIGN AND METHODOLOGY}

\subsection{Study Site Selection}

Selection of the study site to assess SIT, IIT, or combined SIT/IIT application is important for the success of any pilot field trial, and general guidelines for site selection were considered using Mauritius as a case study (Iyaloo et al. 2014). However, due to the differences and uniqueness of any study site, local considerations 
on specific details are needed. For the ideal study site, the following general criteria should be considered:

a) geographically- or ecologically-isolated

b) targeted mosquito species are dominant

c) manageable size for surveillance and monitoring, and

d) good cooperation of the local government and local communities.

The study site selected for the pilot field trial of the SIT/IIT approach in Thailand was located in the Plaeng Yao District of Chachoengsao Province in the eastern part of the country, which is about $120 \mathrm{~km}$ southeast of Bangkok. Three study areas were selected: Nong Satit as the treatment area, Pleang Mai Daeng as the adjacent area, and Nong Sarika as the control area. The distance between the treatment and the control areas was approximately $12 \mathrm{~km}$, whereas the distance between the treatment and the adjacent area was approximately 500-800 meters.

The study areas were located among rice and cassava fields, as well as rubber and other plantations, which formed an ideal partial barrier to the movement of $A e$. aegypti mosquito vectors. The selected study site was considered a typical semi-rural village similar to most other villages in Thailand (Fig. 1). In addition, it met the general criteria for site selection as previously described.
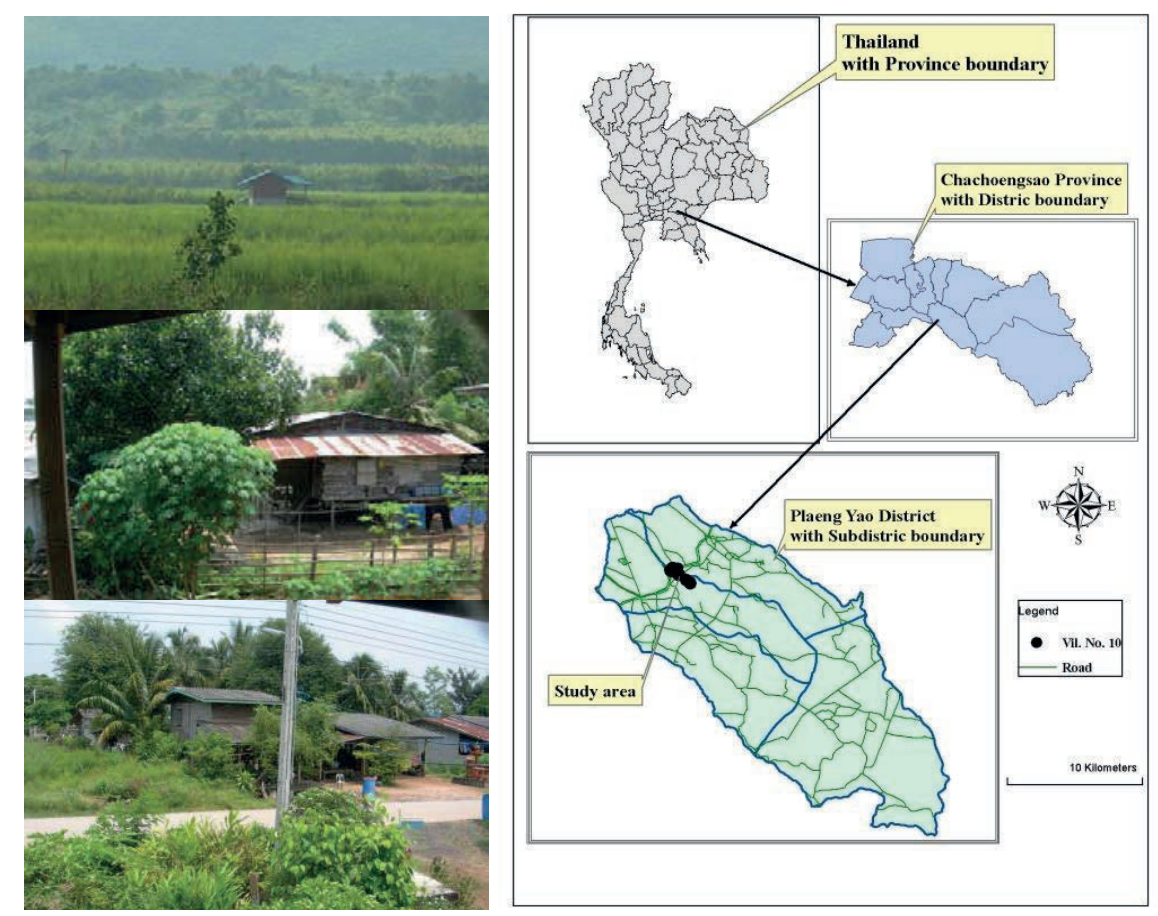

Figure 1. Maps and pictures showing the pilot treatment site located in Nong Satit Village (Village No. 10), Hua Sam Rong Sub-District, Plaeng Yao District, and surrounding areas in Chachoengsao Province, eastern Thailand, where sterile Aedes aegypti male mosquitoes were released for the first time. 


\subsection{Spatial Baseline Data Collection/Mapping of Study Sites}

Spatial data obtained from a geographic information system (GIS), supplemented with 'ground truthing,' were used to characterize spatial distribution and patterns of households located at the study areas. Handheld Global Positioning System (GPS) sets were used to record all houses in the study site, and ArcMap software (ESRI, Redlands, California) was used to develop a GIS map (Chansang and Kittayapong 2007; Kittayapong et al. 2008). This GIS map was useful in determining the sampling households (Fig. 2).

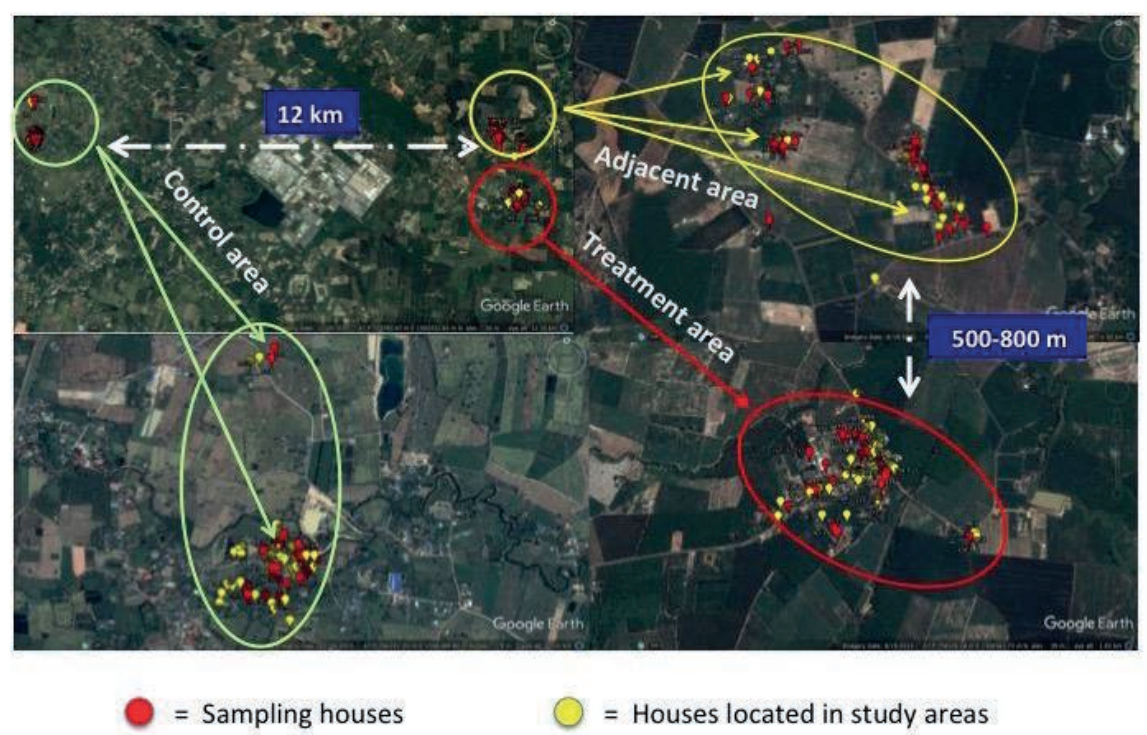

Figure 2. GIS map of the study site (upper left), including the treatment, adjacent, and control areas, and the sampling houses and other houses located in Pleang Yao District, Chachoengsao Province, eastern Thailand.

\subsection{Community Engagement Strategy}

Government authorities were officially informed about the project objectives and methodologies after the project had obtained institutional ethical approval. A community education campaign was organized for the communities in the study area, before implementation of the project, to raise awareness of vector-borne diseases and alternative vector control using combined SIT/IIT. Furthermore, the local leaders were invited to the meetings, and the key message delivered was that male mosquitoes cannot bite and do not feed on blood. In addition, sterile male mosquitoes in screened cages were brought to the community to demonstrate the key message. 
The field release of sterile Ae. aegypti male mosquitoes was emphasized as an additional tool, in combination with other conventional control methods, to prevent vector-borne diseases. The message that other vector control measures, such as breeding container removal, could be applied prior to and during the release of sterile male mosquitoes was also emphasized, so that the community was not under the impression that they were fully protected from vector-borne diseases during the implementation of the sterile male releases. Routine classical vector control measures, i.e. fogging and source reduction, were applied to both treatment and control sites prior to the intervention.

\subsection{Entomological Surveillance and Monitoring}

Mosquito abundance and egg hatch rate of the targeted Ae. aegypti population were estimated in 60 and 90 households, respectively, in the treatment, adjacent, and control areas within the selected study site during the one-year baseline and sixmonth intervention. Mosquito abundance was determined using MosHouse sticky traps and MosVac portable vacuum aspirators (Go Green Co., Ltd., Nakhon Pathom, Thailand). MosHouse sticky traps (Fig. 3) were distributed to 60 households to sample adult mosquitoes in the treatment, adjacent, and control areas.

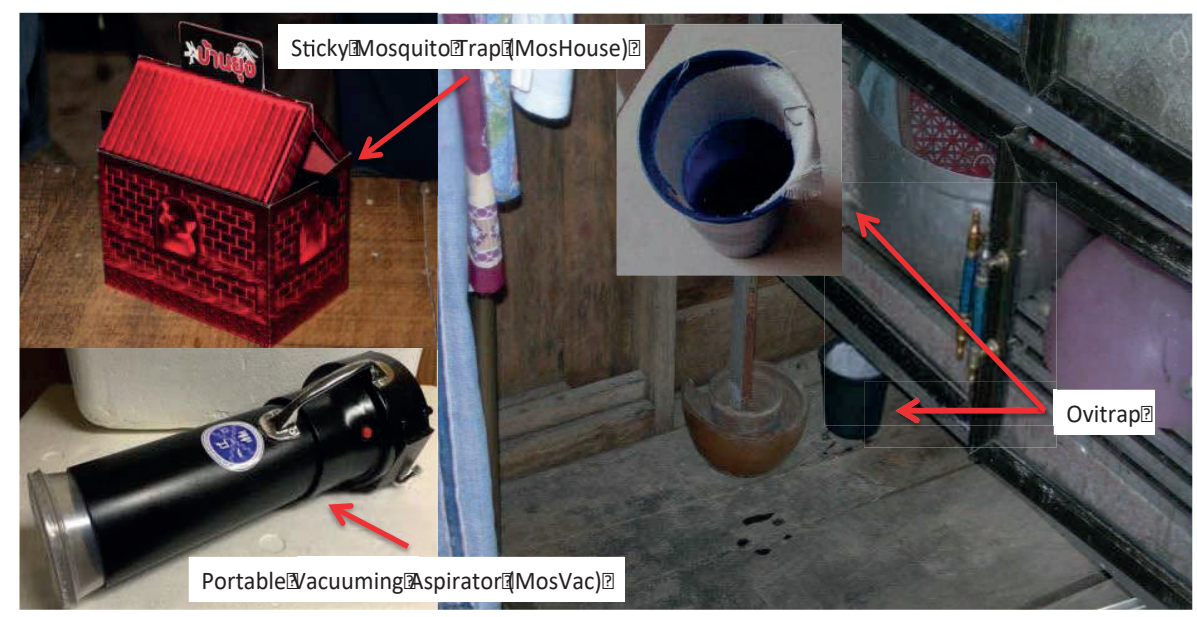

Figure 3. Picture showing the MosHouse sticky trap, the portable mosquito vacuuming aspirator, and the ovitrap that were used for surveillance and monitoring of natural Aedes aegypti populations in the study site.

The MosHouse traps were left in houses for one week before the sticky panels were collected and brought back to the laboratory to identify mosquito species and to determine relative mosquito abundance. In addition, the portable MosVac aspirators (Fig. 3) were used to collect resting mosquitoes in the same 60 households. 
Sampled mosquitoes were killed by freezing and transported to the field laboratory station for species identification. The total number of Ae. aegypti males and females was recorded monthly for a total of two years to determine the dynamics of the different wild Ae. aegypti populations.

Ovitraps (Fig. 3) were distributed in 90 households to allow oviposition and then to collect Ae. aegypti eggs. The filter papers with eggs were collected on a weekly basis and brought back to the laboratory. The eggs that dried on the filter paper were counted and then hatched in water after 2-3 days. The number of eggs hatched was used to determine the sterility of natural Ae. aegypti populations.

\section{EXPERIMENTS REQUIRED BEFORE THE PILOT TRIAL}

\subsection{Rearing of Wolbachia-infected Mosquitoes}

Rearing is a crucial step for SIT/IIT implementation. Genomic adaptation to the massrearing environment, such as reduction in developmental time, life span, dispersal, and stress resistance, as well as early fertility and increased fecundity, is known to occur. This adaptation could make the individuals in the mass-rearing environment significantly different from the wild populations and affect the quality of the released male mosquitoes and hence, the efficacy of SIT/IIT applications (Nikolouli et al. 2018). Moreover, artificially Wolbachia-infected mosquito lines were observed to have increased larval mortality and decreased adult longevity when compared with aposymbiotic ones (Brelsfoard and Dobson 2011). Therefore, a strategy to maintain genetic diversity, biological quality, and competitiveness is required (Nikolouli et al. 2018). A high level of vigilance and consistent standardization of all processes, rearing conditions, and quality control needs to be maintained (Carvalho et al. 2014).

For the SIT/IIT trial in Thailand, mosquitoes were reared at the Center of Excellence for Vectors and Vector-Borne Diseases, Faculty of Science, Mahidol University at Salaya, Nakhon Pathom, Thailand and maintained in aluminium cages $(40 \times 40 \times 40 \mathrm{~cm})$ in a screened insectary at a temperature of $27 \pm 2{ }^{\circ} \mathrm{C}$, a humidity of $75 \pm 2 \%$, and a photoperiod of L12:D12 (Kittayapong et al. 2018). Both male and female mosquitoes had access to a $10 \%$ sucrose solution, and females were fed with pig blood obtained from a qualified slaughterhouse.

The females were offered a blood meal for 3-4 consecutive days after mating using a Hemotek blood-feeding system (Hemotek Ltd., UK). Thereafter, plastic containers with the egg papers were placed inside the cages and were collected after 3-4 days. The eggs were dried and transferred to glass containers with screw-top covers filled with deionized water for egg hatching. After the eggs hatched into first-instar larvae, they were counted manually and transferred into plastic trays $(32 \mathrm{~cm} \times 42 \mathrm{~cm} \times 5 \mathrm{~cm})$, each containing about 2,000 larvae.

The larval diet had the following ingredients: mixed fish meal (Chanpongcharoen Kankaset Supplier, Thailand), pork liver powder, and yeast (Saccharomyces cerevisiae) (Cheese Powder Supplier, Thailand) at a ratio of 5:4:1 respectively. Each tray received $6.5 \mathrm{~g}$ of the diet every day. After 6-7 days, the developed pupae were placed inside plastic containers prior to sex separation. 


\subsection{Sex Separation before Sterilisation}

Population suppression using the combined SIT/IIT approach requires release of a large number of male mosquitoes; therefore, an efficient system to separate the males from the females is essential to release only sterile males into the environment. Many studies have attempted to develop sex separation methods, based on biological, genetic, and transgenic approaches to support the application of the SIT for mosquito control. Sieving techniques were introduced in view of size differences between male and female pupae (Sharma et al. 1972; Bellini et al. 2007).

The development of genetic sexing strains (GSS), as well as other sex separation strategies, is currently under development and/or refinement, but none of them have so far succeeded in eliminating all females in order to achieve male-only releases for large-scale SIT or other applications (Benedict et al. 2009; Papathanos et al. 2009, 2018; Gilles et al. 2014).

Larval-pupal glass separators (Model 5412, John W. Hock, Co., Ltd., Gainesville, Florida, USA) were used to mechanically separate male and female pupae into different layers. The female pupae are larger in size and are collected in an upper layer between the two adjusted glass plates, while male pupae are drained into a receiving container placed below. Water circulation is supplied all along the process to push and wash the pupae down into the container. The female pupae are eventually flushed into a second receiving container, and the cycle of sex separation is complete.

In the experiments of sex separation, one litre of water that contained about 1500 to 2000 mixed male and female pupae were introduced each cycle into the system. One cycle took on average between 2 and 5 minutes, but it could take longer if the sample was mixed with larvae. After counting, the male pupae were transferred into a plastic cup and transported to the radiation source.

\subsection{Appropriate Irradiation Dose for Male Sterilisation}

Appropriate irradiation doses are different for different species of mosquitoes. Our preliminary studies showed that when Wolbachia-infected Ae. aegypti mosquitoes were irradiated at the pupal stage, an irradiation dose of 50 Gy was sufficient to obtain complete sterility in females, while males were fully sterilized with a dose of $70 \mathrm{~Gy}$. Wolbachia-infected male pupae irradiated with 50 Gy could still produce some viable eggs when mated as adults with non-irradiated Wolbachia-infected females, the average percentage of egg hatch being $8 \%$. However, egg hatch was zero when Wolbachia-infected males and females were irradiated with 70 Gy and then mated with non-irradiated Wolbachia-infected females and males, respectively (Kittayapong et al. 2018).

Since the Wolbachia-infected Ae. aegypti mosquitoes used in the pilot SIT/IIT trial in Thailand did not express complete CI (Ruang-areerate and Kittayapong 2006; Kittayapong et al. 2019), the complete sterility in these experiments was obtained through appropriate irradiation doses. If Wolbachia strains expressing strong CI are used, lower irradiation doses can be applied in order to obtain complete sterility of Ae. aegypti mosquitoes. 


\subsection{Mating Competitiveness and Release Ratio}

Mating competitiveness of sterile male mosquitoes needs to be assessed before implementing a pilot SIT/IIT trial. In the past, many SIT trials were not successful in reducing natural mosquito populations due to the low competitiveness of sterile males after they were irradiated with too high doses (Dame et al. 2009). The advantage of the combined SIT/IIT approach is that lower dose radiation can be applied, as the sterility can also be induced in Ae. aegypti mosquitoes by the CI property of Wolbachia bacteria.

In the cage study, under controlled laboratory conditions, sterile Ae. aegypti males were evaluated for their mating competitiveness with wild males and females at different ratios (Fig. 4). Results indicated that a ratio of 10:1:1 or above was effective, as it reduced egg hatch significantly. The hatched eggs/total eggs of the 10:1:1 ratio experimental group was $3 / 619(0.27 \pm 0.65 / 103.17 \pm 9.09)$. Complete sterility was observed with no egg hatch at a ratio of $20: 1: 1$. Therefore, ratios between 10:1:1 and 20:1:1 were determined to be the optimal release ratios for the mass-reared sterile $A e$. aegypti males, as they could compete with the wild males and induce near complete or complete sterility in the wild females. Our results also indicated that an irradiation dose of 70 Gy did not reduce mating competitiveness of the irradiated Ae. aegypti males (Kittayapong et al. 2019). In conclusion, our laboratory experiments demonstrated no significant difference in competitiveness of sterile males when compared to wild ones.

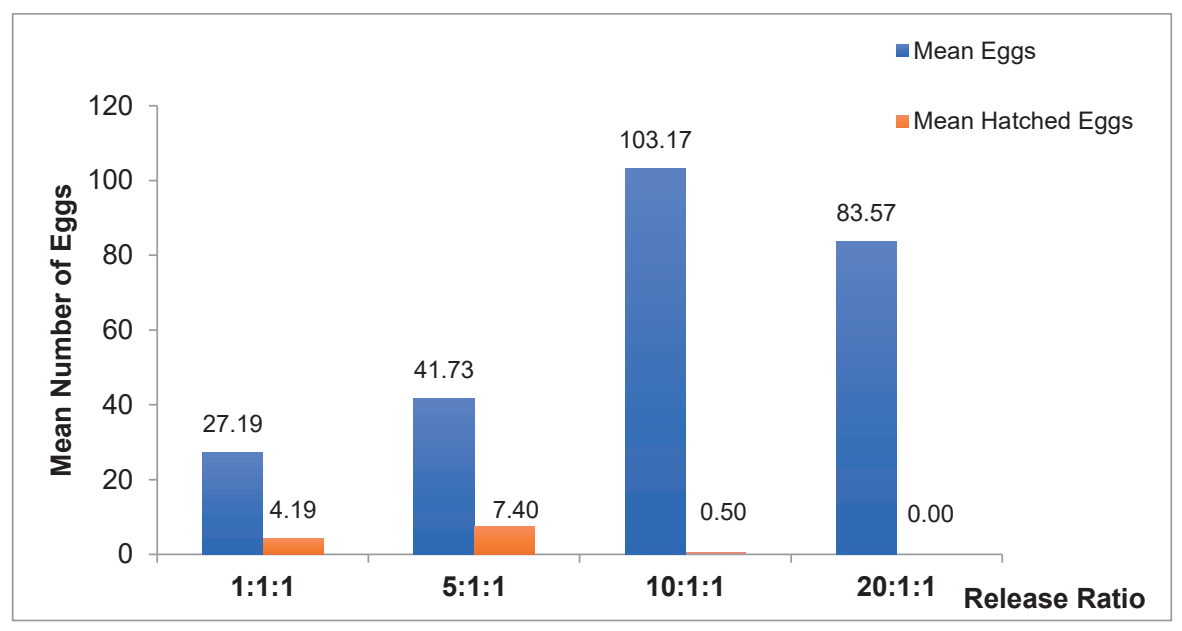

Figure 4. Mating competitiveness of sterile Aedes aegypti males at different sterile male: wild male: wild female release ratios in cages under controlled laboratory conditions (modified from Kittayapong et al. 2019). 


\subsection{Survival and Longevity of Sterile Males}

Survival and longevity of the released sterile male mosquitoes are other parameters that may have an impact on the success of a SIT/IIT programme. The longer the sterile males can live, the higher the probability of mating with a wild female. In nature, both wild and sterile males should have shorter life spans than those kept under optimal controlled conditions. The same applies to wild and sterile females.

In our baseline experiments, carried out under controlled laboratory conditions, we observed that there was no significant difference in longevity between wild and sterile Ae. aegypti males (Fig. 5). On average, the wild males survived for $23.3 \pm 0.9$ days, while the sterile males survived for $23.8 \pm 12.1$ days. However, wild females lived significantly longer than sterile females, i.e. an average life span of $29.6 \pm 1.0$ days and $18.5 \pm 9.8$ days, respectively $(p=0.000)$ (Kittayapong et al. 2018).

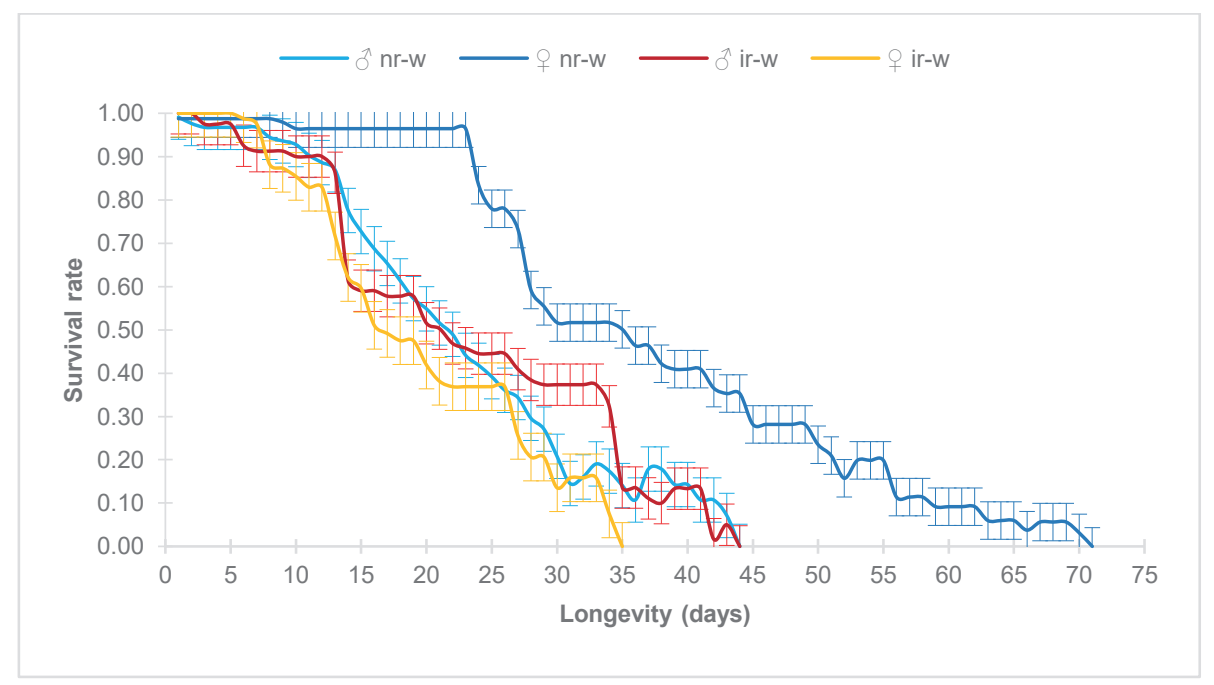

Figure 5. Mean longevity and survival rate of non-irradiated ( $n r)$ and irradiated (ir) Wolbachia-infected Aedes aegypti male and female mosquitoes, after being sex-separated by using larval-pupal glass separators (modified from Kittayapong et al. 2018).

\section{EXPERIENCE AND LESSONS LEARNED FROM THE PILOT TRIAL}

\subsection{Community/Public/Stakeholder Engagement}

Local stakeholders were identified. Engagement of local government authorities was initiated and followed up. Local government authorities coordinated with community leaders to initiate community engagement and facilitate community participation in implementing further on-site research activities, such as entomological surveillance and sterile male mosquito delivery using local health volunteers. Public education through media was carried out to raise awareness regarding vector-borne diseases and potential risk reduction using an alternative SIT/IIT approach. 
From our experience, engagement of the community and public was more effective when it was initiated during times of epidemics. Our public engagement was initiated when both dengue and Zika were epidemic and people were aware of the consequences. Furthermore, we took advantage of related public events to draw the attention of the public. The first open release of sterile male mosquitoes was carried out on ASEAN Dengue Day, when the Ministry of Public Health of Thailand was an official host of regional activities (Fig. 6); hence representatives of many countries were present and witnessed the opening ceremony and first release of sterile males to fight dengue in Thailand.

In this pilot project, the general public was engaged through several national media reports, and TV news and radio programmes. A total of 109 media items, including a documentary, international news, national news, national radio, newspaper and online articles, and TV shows, were produced from January 2016 to February 2018 for public education of the sterile male release in Thailand.

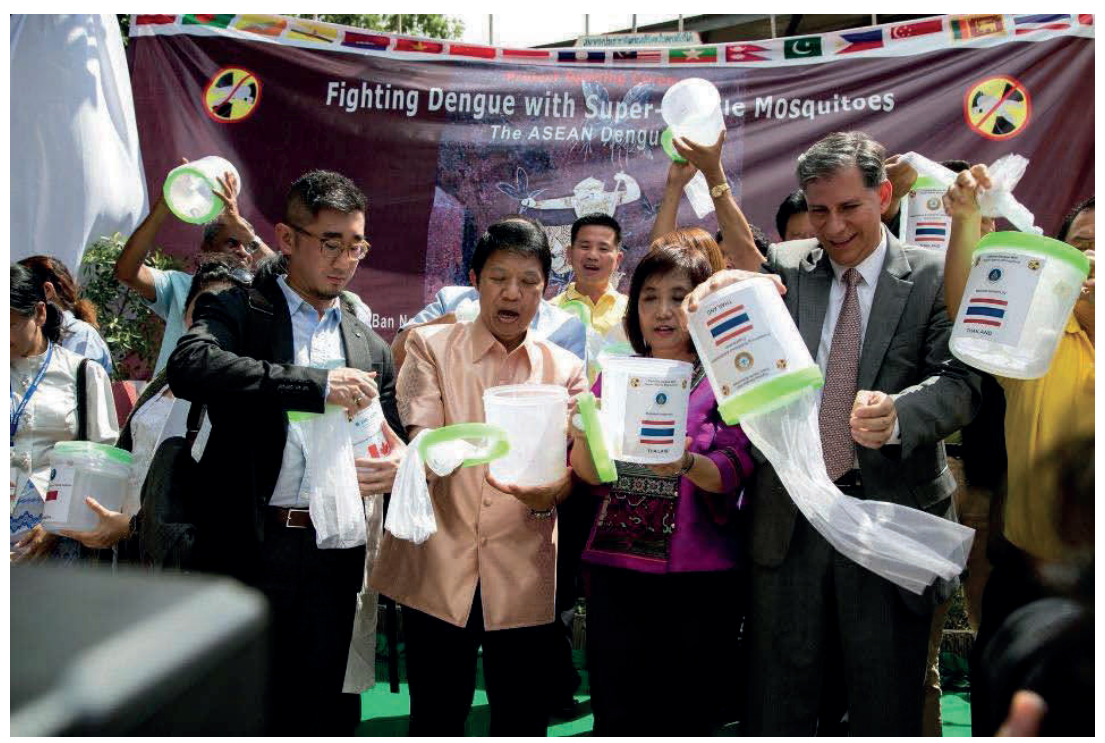

Figure 6. Picture showing the first release of sterile Aedes aegypti male mosquitoes on ASEAN Dengue Day at the study site in Pleang Yao District, Chachoengsao Province, eastern Thailand.

A high number of views and sharing on social media, with the online articles published by reliable media publishers, was experienced (Kittayapong et al. 2019). Therefore, social media is an interesting additional channel to be used to communicate key messages to most of the general public. In addition, TV shows and documentaries on the topic of controlling the dengue vector through the SIT/IIT approach gained a lot of public attention in Thailand. 


\subsection{Handling and Transport of Sterile Mosquitoes for Open Field Release}

Ideally, the radiation source used to sterilize the male mosquitoes should be in the same place as the rearing facility, while the subsequent transport to the release sites could be either as pupae or adults. In our case, male pupae had to be transported to a laboratory located $120 \mathrm{~km}$ from the rearing site for the irradiation treatment. Wolbachia-transinfected Ae. aegypti male mosquitoes were produced in the screened insectary at the Center of Excellence for Vectors and Vector-Borne Diseases, Faculty of Science, Mahidol University, Salaya Campus, Nakhon Pathom, and were then transported to the Thailand Institute of Nuclear Technology (TINT) in Nakhon Nayok for sterilisation at the pupal stage of 1-2 days old using a ${ }^{60} \mathrm{Co}$ source.

Sterile male pupae were transported weekly to the study sites using temperaturecontrolled containers. Our preliminary experiments showed $100 \%$ survival of the chilled sterile male pupae at temperatures between $8-12^{\circ} \mathrm{C}$ for up to 6 hours (Kittayapong et al. unpublished data).

Even though sterilisation at the pupal stage was shown to have no impact on mating competitiveness and the longevity of irradiated mosquitoes, it would be more practical to irradiate adult mosquitoes and then use the same containers for release. This would be less time-consuming, as the adult mosquitoes would not have to be transferred to the different release containers. Further experiments are needed to decide on the best temperature and container for chilling and transporting adults.

\subsection{Management and Implementation of Sterile Male Release}

A simple field laboratory station was set up in the city of Chachoengsao, $20 \mathrm{~km}$ from the release site in Plaeng Yao District. The research team worked with local workers to transfer male pupae to the release containers for adult emergence. Sterile Ae. aegypti males were provided with a $10 \%$ sucrose solution as food source after emergence for at least one day. They were then transported to the selected study sites, and the public health volunteers released the sterile mosquitoes at a rate of 100-200 per household per week. A total number of 437980 sterile Ae. aegypti males, ranging from 9000 to 25000 males per week, were released. The weekly releases were carried out only in the treatment area of the study site for a period of 24 weeks.

In this pilot trial, ULV fogging was used by local government staff to reduce the natural populations of Ae. aegypti to low densities before the releases of sterile male Ae. aegypti were initiated. The vector control activities by local government staff were conducted in both treatment and control areas. Public health volunteers provided assistance with the delivery of the sterile male mosquitoes to their respective households (Fig. 7). 


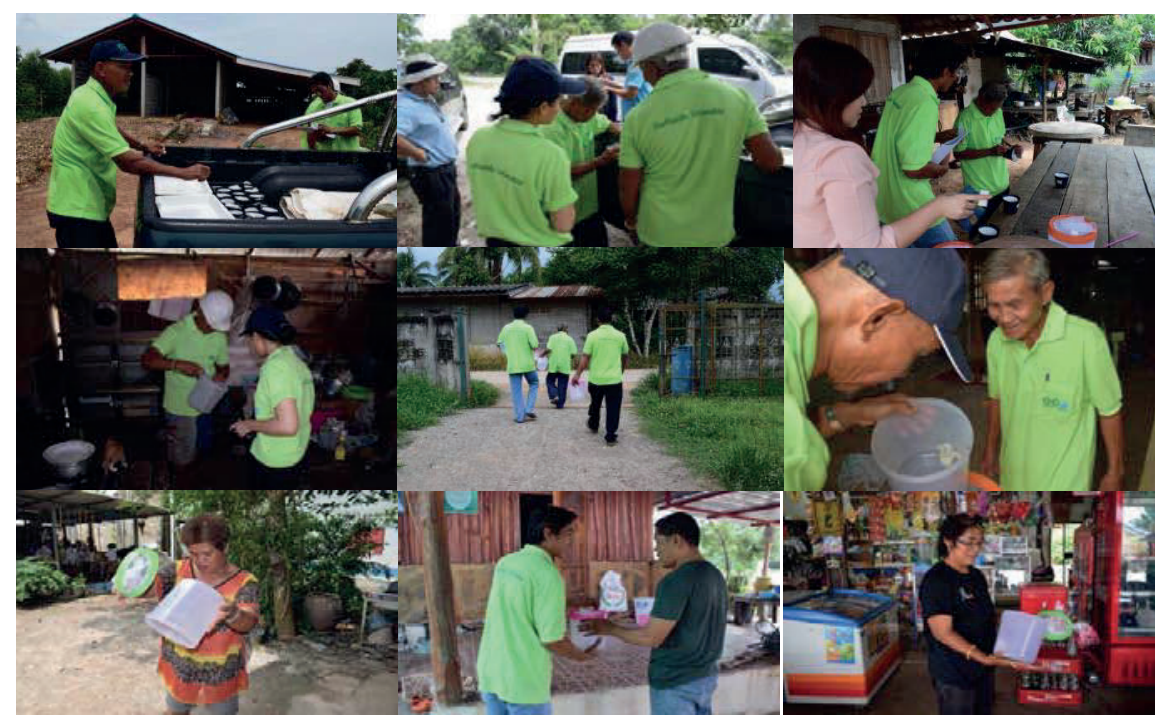

Figure 7. Pictures showing activities related to the release of sterile males in the treatment community in Plaeng Yao District, Chachoengsao Province, eastern Thailand, by the health volunteers and homeowners under the supervision of the research team.

From our experience, frequent entering of private property caused some reluctance of a few homeowners to continue to cooperate. A visit of our staff together with the public health volunteers to these few houses was necessary to keep them cooperating. Therefore, the future planning of the release strategy should consider reducing disturbance of the privacy of homeowners. Open release using drones is recommended, especially in urban and crowded communities where intrusion into households is difficult. However, successful application of drone releases of sterile mosquitoes will need authorized and skilled operation.

\subsection{Surveillance and Monitoring of Sterile Mosquitoes after Release}

Both MosHouse sticky traps and MosVac portable vacuuming aspirators were used in the households of the study site for collecting both male and female mosquitoes of various species. MosHouse traps seemed to be more efficient in collecting Ae. aegypti females, while resting males were collected in higher numbers by using the MosVac portable vacuuming aspirators. When comparing the relative abundance of the Ae. aegypti mosquito populations, the average number of Ae. aegypti females sampled in the treatment area significantly decreased $(p<0.05)$ when compared to those in the control area, while those of males were not significantly different $(p>0.05)$, even though a large number of sterile males were released during the six-month intervention period in the treated areas (Table 1). 
Table 1. Comparison of the mean numbers of Aedes aegypti males and females collected by using MosHouse sticky traps and MosVac portable mosquito vacuuming

aspirators in the treatment, adjacent, and control areas in Plaeng Yao District, Chachoengsao Province, eastern Thailand during the six-month intervention period (modified from Kittayapong et al. 2019)

\begin{tabular}{|c|c|c|c|c|c|c|c|}
\hline Variable & $\begin{array}{c}\text { No. } \\
\text { House }\end{array}$ & $\begin{array}{c}\text { No. } \\
\text { Mos- } \\
\text { House } \\
\text { traps }\end{array}$ & $\begin{array}{c}\text { No. positive } \\
\text { household } \\
\text { (Mean } \pm \\
\text { SD) }\end{array}$ & $\begin{array}{c}\text { Total } \\
\text { mosquitoes } \\
(\text { Mean } \pm \text { SD) }\end{array}$ & $\begin{array}{l}\text { Odds } \\
\text { Ratio } \\
\text { (total mos- } \\
\text { quitoes) }\end{array}$ & $95 \% \mathrm{CI}$ & $P$ \\
\hline \multicolumn{8}{|l|}{ Males } \\
\hline Control & 20 & 20 & $15.83 \pm 1.6$ & $\begin{array}{c}193 \\
(32.17 \pm 4.07)\end{array}$ & 1 & & \\
\hline Adjacent & 20 & 20 & $10.00 \pm 3.4$ & $\begin{array}{c}92 \\
(15.33 \pm 6.31)\end{array}$ & 0.263 & $0.149-0.464$ & $0.000^{*}$ \\
\hline Treatment & 20 & 20 & $16.50 \pm 2.9$ & $\begin{array}{c}137 \\
(22.83 \pm 6.55)\end{array}$ & 1.242 & $0.651-2.373$ & 0.511 \\
\hline \multicolumn{8}{|l|}{ Females } \\
\hline Control & 20 & 20 & $17.00 \pm 3.2$ & $\begin{array}{c}185 \\
(30.83 \pm 7.05)\end{array}$ & 1 & & \\
\hline Adjacent & 20 & 20 & $4.83 \pm 1.83$ & $\begin{array}{c}35 \\
(5.83 \pm 2.64)\end{array}$ & 0.056 & $0.029-0.108$ & $0.000^{*}$ \\
\hline Treatment & 20 & 20 & $2.67 \pm 1.75$ & $\begin{array}{c}16 \\
(2.67 \pm 1.75)\end{array}$ & 0.027 & $0.013-0.056$ & $0.000^{*}$ \\
\hline
\end{tabular}

${ }^{*}$ Significant difference at $p<0.05$

It is possible that Ae. aegypti males mostly rested outside households where trapping and vacuuming activities took place (Table 1). The lower numbers of $A e$. aegypti females in the treatment area compared to the control area indicate the effect of the sterile male releases that produced sterility in the wild Ae. aegypti females, resulting in a reduction in the numbers of Ae. aegypti female populations in nature by up to $97.30 \%$ (Fig. 8 ).

MosHouse sticky trap and MosVac portable vacuuming aspirators can be employed as tools for monitoring SIT, IIT, or combined SIT/IIT interventions, especially in view of their low cost and uncomplicated deployment. The advantage of the MosHouse traps as compared with the MosVac aspirators is that they can be placed either inside or around households without disturbing the homeowners. In addition, large numbers of the low-cost MosHouse sticky traps can be distributed in different locations in the study areas, resulting in better estimates of natural $A e$. aegypti populations, compared to using a few high-cost traps placed in only a few locations.

As Ae. aegypti mosquitoes are more domestic, placing a few traps in a few locations could lead to a biased estimation of the total natural populations in the study areas. However, additional methods for collecting mosquitoes could be applied in combination to obtain more reliable data sets for entomological evaluation. 
a
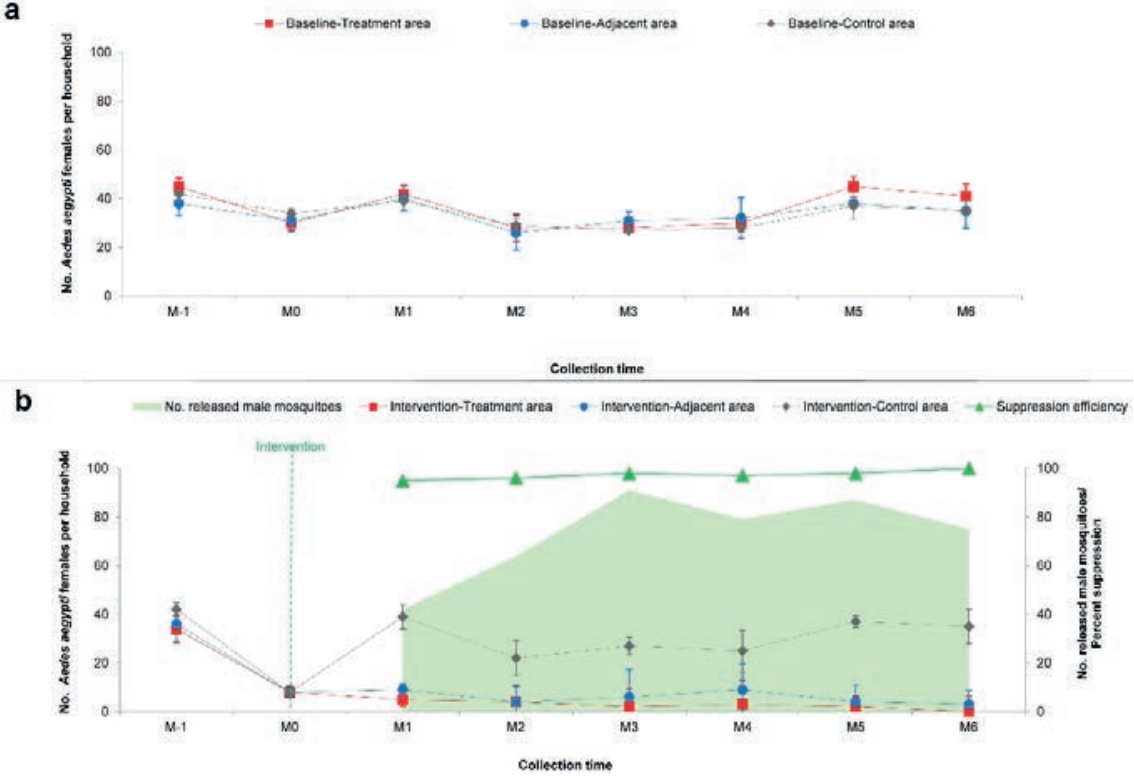

Figure 8. Mean numbers of Aedes aegypti female mosquitoes collected per households in Pleang Yao District, Chachoengsao Province, Thailand during the baseline (Fig. 8a) and during the intervention (Fig. 8b) periods. Percent suppression efficiency in relation to the number of released sterile males per month is demonstrated in Fig. $8 b$ (modified after

Kittayapong et al. 2019).

Weekly ovitrap data showed that the overall mean egg hatch was lowest in the treatment area, confirming the effectiveness of the sterile male release. The mean egg hatch for the treatment, adjacent, and control areas were $0.20 \pm 0.10,0.24 \pm 0.14$, and $0.41 \pm 0.08$ respectively; while those for the second twelve weeks were $0.18 \pm 0.09$, $0.25 \pm 0.16$, and $0.54 \pm 0.11$ respectively (Fig. 9).

There was a significant difference $(p<0.05)$ in mean egg hatch between the first and the second twelve weeks of sterile male releases (Table 2 and Table 3 ). The released sterile males seemed to show positive effects in reducing hatched eggs in the natural Ae. aegypti mosquito populations, in both the treatment area and the adjacent area, when compared to the households monitored in the control area (Fig. 9).

Except for a few outliers, egg hatch decreased to zero or near zero in most of the households monitored in the treatment area and adjacent area (Table 2). Since very low numbers of Ae. albopictus were found in this study area, especially in households, we could assume that the unhatched eggs were mostly from Ae. aegypti (Table 2 and Table 3). 


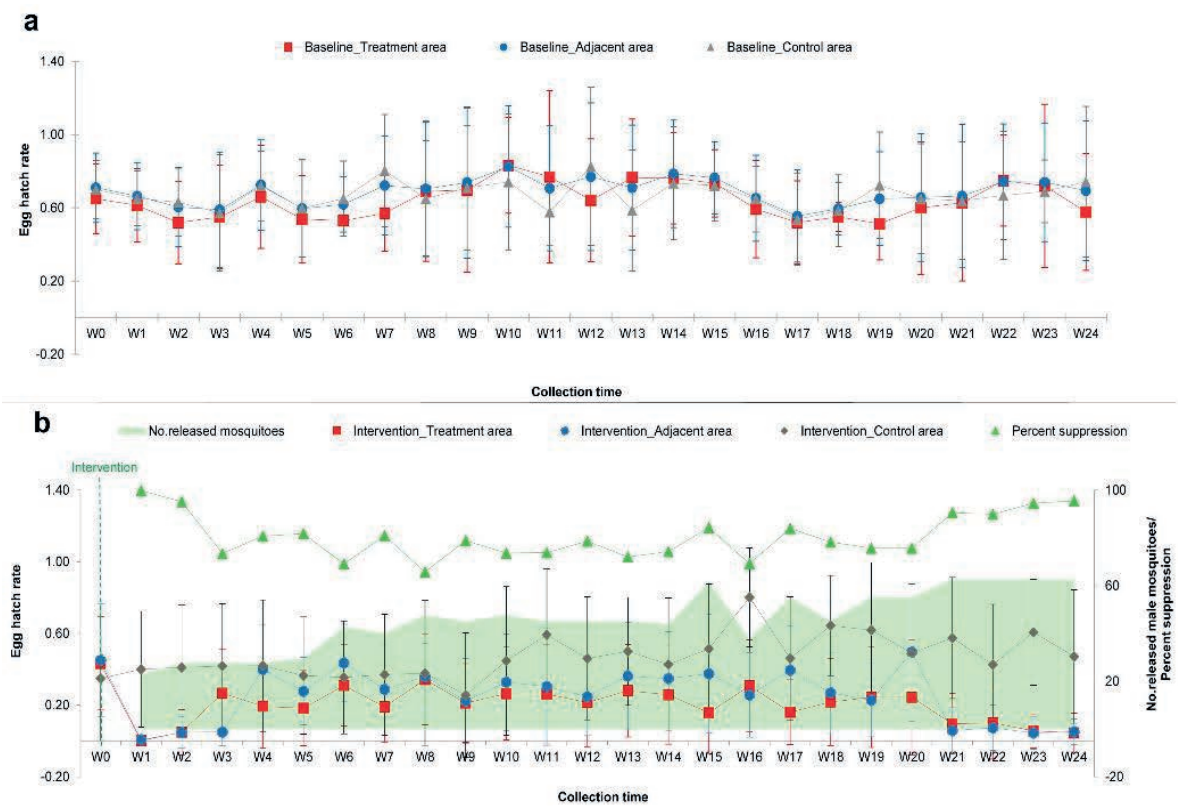

Figure 9. Mean egg hatch rate of natural Aedes aegypti mosquito populations over time in the treatment, adjacent, and control areas of the study sites during the

baseline (a) and during the intervention (b) periods. Percent suppression efficiency in relation to the number of released sterile males per week is demonstrated in

Fig. $9 b$ (modified after Kittayapong et al. 2019).

\subsection{Quality Control of Sterile Mosquito Production}

Quality control to test for sterility of irradiated Wolbachia-infected Ae. aegypti males can be done through mating studies between mass-produced sterile males and untreated females from established mosquito colonies with no Wolbachia infection, originally collected from the same study site. Zero or near zero egg hatch was expected for each production lot. Mating tests performed during the 24-week open field trial in Plaeng Yao District, Chachoengsao Province, eastern Thailand indicated that the average numbers of hatched eggs in most production lots were quite low, i.e. $1.04 \pm 2.18$, which demonstrated that the irradiated Wolbachia-infected male mosquitoes were highly sterile (Kittayapong et al. 2018).

In addition, Wolbachia detection by PCR was conducted in 40 sterile Ae. aegypti males sampled from each of the production lots, and the results showed that the mean percentage of Wolbachia infection was $50.21 \pm 0.49 \%$ in released males (Kittayapong et al. 2019). 
Table 2. Progressive egg hatch rate of Aedes aegypti mosquitoes in households in the treatment area, before and after 12 and 24 weeks of releases, when compared to those in households in the adjacent and the control areas

\begin{tabular}{|c|c|c|c|c|c|c|c|c|c|}
\hline \multirow[t]{3}{*}{ No. } & \multicolumn{3}{|c|}{ Before releases } & \multicolumn{3}{|c|}{ After 12 weeks of releases } & \multicolumn{3}{|c|}{ After 24 weeks of releases } \\
\hline & \multicolumn{3}{|c|}{ W0 } & \multicolumn{3}{|c|}{ W12 } & \multicolumn{3}{|c|}{ W24 } \\
\hline & $\begin{array}{c}\text { Control } \\
\text { area }\end{array}$ & $\begin{array}{c}\text { Adjacent } \\
\text { area }\end{array}$ & $\begin{array}{c}\text { Treatment } \\
\text { area }\end{array}$ & $\begin{array}{c}\text { Control } \\
\text { area }\end{array}$ & $\begin{array}{c}\text { Adjacent } \\
\text { area }\end{array}$ & $\begin{array}{c}\text { Treatment } \\
\text { area }\end{array}$ & $\begin{array}{c}\text { Control } \\
\text { area }\end{array}$ & $\begin{array}{c}\text { Adjacent } \\
\text { area }\end{array}$ & $\begin{array}{c}\text { Treatment } \\
\text { area }\end{array}$ \\
\hline 1 & 0.60 & 0.00 & 0.00 & 0.11 & 0.29 & 0.10 & 1.00 & 0.00 & 0.00 \\
\hline 2 & 0.00 & 0.25 & 0.28 & 0.37 & 0.00 & 0.00 & 0.43 & 0.00 & 0.00 \\
\hline 3 & 0.09 & 0.81 & 0.38 & 0.59 & 0.00 & 0.44 & 0.80 & 0.00 & 0.16 \\
\hline 4 & 0.29 & 0.23 & 0.64 & 0.87 & 0.00 & 0.00 & 0.27 & 0.05 & 0.38 \\
\hline 5 & 0.39 & 0.34 & 0.00 & 0.26 & 0.46 & 0.57 & 0.68 & 0.00 & 0.00 \\
\hline 6 & 0.00 & 0.52 & 0.48 & 0.43 & 0.00 & 0.02 & 0.00 & 0.09 & 0.00 \\
\hline 7 & 0.00 & 0.00 & 0.03 & 0.96 & 0.19 & 0.25 & 0.15 & 0.00 & 0.00 \\
\hline 8 & 0.74 & 0.68 & 0.10 & 0.36 & 0.17 & 0.00 & 0.60 & 0.00 & 0.00 \\
\hline 9 & 0.00 & 0.94 & 0.67 & 1.00 & 0.34 & 0.00 & 0.32 & 0.04 & 0.07 \\
\hline 10 & 0.73 & 0.95 & 0.87 & 0.81 & 0.28 & 0.00 & 0.77 & 0.00 & 0.00 \\
\hline 11 & 0.22 & 0.52 & 0.64 & 0.00 & 0.00 & 0.26 & 0.44 & 0.00 & 0.07 \\
\hline 12 & 0.94 & 0.31 & 0.39 & 1.00 & 0.16 & 0.54 & 0.44 & 0.18 & 0.00 \\
\hline 13 & 0.67 & 0.26 & 0.18 & 0.56 & 0.08 & 0.00 & 0.00 & 0.15 & 0.00 \\
\hline 14 & 0.94 & 0.20 & 0.39 & 0.47 & 0.00 & 0.61 & 0.20 & 0.00 & 0.04 \\
\hline 15 & 0.00 & 0.35 & 0.00 & 0.94 & 0.84 & 0.39 & 0.00 & 0.01 & 0.00 \\
\hline 16 & 0.12 & 0.94 & 0.00 & 0.00 & 0.22 & 0.00 & 0.00 & 0.00 & 0.00 \\
\hline 17 & 0.00 & 0.09 & 0.00 & 0.31 & 0.67 & 0.00 & 0.50 & 0.00 & 0.00 \\
\hline 18 & 0.94 & 0.53 & 0.00 & 0.18 & 0.18 & 0.50 & 0.30 & 0.14 & 0.10 \\
\hline 19 & 0.19 & 0.28 & 0.74 & 0.95 & 0.29 & 0.00 & 0.94 & 0.00 & 0.00 \\
\hline 20 & 0.09 & 0.80 & 0.37 & 0.94 & 0.59 & 0.50 & 0.60 & 0.03 & 0.46 \\
\hline 21 & 0.00 & 0.20 & 0.74 & 0.47 & 0.42 & 0.23 & 0.00 & 0.13 & 0.00 \\
\hline 22 & 0.94 & 0.00 & 0.21 & 0.17 & 0.26 & 0.50 & 1.00 & 0.07 & 0.00 \\
\hline 23 & 0.00 & 0.23 & 0.00 & 0.45 & 0.14 & 0.16 & 0.00 & 0.27 & 0.00 \\
\hline 24 & 0.21 & 0.62 & 0.29 & 0.33 & 0.26 & 0.84 & 0.70 & 0.00 & 0.07 \\
\hline 25 & 0.13 & 0.47 & 0.00 & 0.34 & 0.04 & 0.00 & 1.00 & 0.15 & 0.00 \\
\hline 26 & 0.00 & 0.82 & 0.00 & 1.00 & 0.39 & 0.00 & 0.00 & 0.07 & 0.00 \\
\hline 27 & 0.00 & 0.80 & 0.71 & 1.00 & 0.00 & 0.26 & 0.31 & 0.00 & 0.00 \\
\hline 28 & 0.00 & 0.00 & 0.55 & 0.00 & 0.37 & 0.00 & 1.00 & 0.03 & 0.00 \\
\hline 29 & 0.00 & 0.41 & 0.00 & 0.31 & 0.49 & 0.00 & 0.10 & 0.00 & 0.00 \\
\hline 30 & 0.33 & 0.94 & 0.00 & 0.00 & 0.25 & 0.25 & 1.00 & 0.13 & 0.00 \\
\hline
\end{tabular}

High hatch rate $(>0.50)$

Moderate hatch rate (0.25-0.50)

Low hatch rate (0.01-0.24)

Zero hatch rate 
Table 3. Statistical analysis of the egg hatch rate of Aedes aegypti during the sixmonth (weeks 1-12 and weeks 13-24) intervention period in the treatment, adjacent, and control areas located in Plaeng Yao District, Chachoengsao Province, eastern Thailand (modified from Kittayapong et al. 2019)

\begin{tabular}{|c|c|c|c|c|c|c|c|}
\hline Variable & $\begin{array}{c}\text { No. } \\
\text { house }\end{array}$ & $\begin{array}{c}\text { No. } \\
\text { ovitrap }\end{array}$ & $\begin{array}{c}\text { No. positive } \\
\text { household } \\
\text { (Mean } \pm \\
\text { SD) }\end{array}$ & $\begin{array}{l}\text { Egg hatch } \\
\text { rate } \\
(\text { Mean } \pm \\
\text { SD) }\end{array}$ & $\begin{array}{c}\text { Odds } \\
\text { Ratio } \\
\text { (Egg } \\
\text { hatch } \\
\text { rate) }\end{array}$ & $95 \% \mathrm{CI}$ & $P$ \\
\hline \multicolumn{8}{|l|}{ W1-W12 } \\
\hline Control & 30 & 60 & $22.00 \pm 0.43$ & $0.41 \pm 0.08$ & 1 & & \\
\hline Adjacent & 30 & 60 & $24.50 \pm 0.39$ & $0.24 \pm 0.14$ & 1.620 & $0.679-3.862$ & 0.277 \\
\hline Treatment & 30 & 60 & $18.00 \pm 0.50$ & $0.20 \pm 0.10$ & 0.545 & $0.252-1.179$ & 0.123 \\
\hline \multicolumn{8}{|l|}{ W13-W24 } \\
\hline Control & 30 & 60 & $24.50 \pm 0.39$ & $0.54 \pm 0.11$ & 1 & & \\
\hline Adjacent & 30 & 60 & $19.00 \pm 0.47$ & $0.25 \pm 0.16$ & 0.388 & $0.168-0.897$ & $0.027^{*}$ \\
\hline Treatment & 30 & 60 & $12.50 \pm 0.48$ & $0.18 \pm 0.09$ & 0.160 & $0.070-0.368$ & $0.000^{*}$ \\
\hline
\end{tabular}

Our experience confirmed that an irradiation dose of $70 \mathrm{~Gy}$ is optimal to induce sterility in Ae. aegypti male mosquitoes. In our experiments, we also observed that Ae. aegypti females were more radio-sensitive and that a treatment with 50 Gy was sufficient to obtain complete female sterility. Therefore, accidentally released irradiated Wolbachia-infected Ae. aegypti females in our field trial, if any, were fully sterile after exposure to $70 \mathrm{~Gy}$, and there was no danger of further propagation or Wolbachia establishment in the target population.

\subsection{Female Contamination during SIT/IIT Implementation}

Sustainable suppression of Ae. aegypti populations by integration of the SIT/IIT depends on the release of only sterile males. Hence, sex separation of mass-produced male and female mosquitoes is an important step, as female contamination could lead to an increase in disease transmission, although it is unlikely due to their Wolbachia infection. Inspection of female contamination was carried out weekly during the twenty-four weeks of the pilot field release of sterile Ae. aegypti males at the selected study site in Plaeng Yao District, Chachoengsao Province, eastern Thailand. Our results indicate a low percentage of female contamination among sterile males, i.e. $0.06 \pm 0.10 \%$, when Ae. aegypti pupae were separated through mechanical larvalpupal glass separators. Therefore, at least $99 \%$ of sterile males were purely separated from females (Kittayapong et al. 2018).

In this study, we also observed a significant difference in the percentage of female contamination during the first and the second 12 -week periods of sterile male releases, i.e. $0.10 \pm 0.13 \%$ vs $0.02 \pm 0.02 \%(p<0.05)$. The percentage of female contamination was remarkably reduced in the second twelve weeks of intervention. This was most likely due to the increasing skills of the technicians operating the mechanical sex 
separation machine. As such, we recommend hands-on training for operating technicians before project implementation to obtain a high efficiency in the manual sex separation process, and hence achieve the lowest possible female contamination during sterile male release.

\subsection{Impact of SIT/IIT on the Environment and Ecosystem}

The SIT/IIT approach for Ae. aegypti mosquito vectors was implemented using a twostep sterilisation process, combining the Wolbachia-induced IIT with the SIT using radiation to obtain sterile males. When these sterile males are systematically released into the target area, they can induce sterility in wild females after mating. Mated females lay eggs that cannot hatch, resulting in significant reduction in natural Ae. aegypti populations and subsequently, an "assumed" reduction in disease incidence that needs to be verified. In general, the SIT/IIT intervention is assessed to have little or no impact on the environment for the following reasons:

1. Mosquitoes released into the environment are irradiated males to ensure sterility. Also, any accidentally released females do not transmit disease if they are infected with pathogen-resistant Wolbachia strains. Thus, using both CI, the property of the Wolbachia endosymbiont that induces sterility in wild females, and radiation to sterilise the Wolbachia-infected mosquitoes, makes sure that they cannot become established in nature. These sterile mosquitoes have shorter life spans due to either the Wolbachia life-shortening effect or irradiation effect, and they will not survive in the natural environment longer than 2-3 weeks after release (Kittayapong et al. 2019). However, this means that the mosquitoes need to be released systematically into the target area to obtain the population reducing effect. Once the native mosquito population is at a low level, fewer sterile males can be released. In view of their short life span, there should be no residual mosquitoes left in the environment a few weeks after termination of the release activities.

2. As this method is species-specific, interfering only with the reproduction of the target population, it has no impact on beneficial insects or any other animals or humans, unlike chemical spraying which impacts the environment, affects non-target organisms, and can leave some residues.

3. The ecosystem will obviously experience a reduction of the Ae. aegypti vectors, and hence a reduction of available food for animals that feed on them. However, as there are over hundred species of mosquitoes in the tropical zone, together with the low biomass of the target population, other mosquito species should be able to serve in the food chain for some predators; therefore, the impact on the ecosystem in this regard should be very low or negligible.

Risk assessment on the use of Wolbachia for controlling mosquito vectors, both in terms of replacement and suppression approaches, was evaluated in the past, and a very low risk for the environment was reported (Popovici et al. 2010, Murray et al. 2016; NEA 2016). 


\section{CONCLUSIONS}

The successful development and implementation of an operational SIT/IIT programme depends on several factors, and therefore these programmes require extensive and thorough planning based on available knowledge of the genetics, biology, and ecology of the target insect species. These include establishing and maintaining a Wolbachia-infected colony of the target species, understanding the field conditions and target population dynamics, assuring community participation, and assessing the potential side effects on humans and the environment.

The SIT is an environment-friendly method. Being species-specific and leaving no toxic residues, it has only minimal or no non-target impact, which has been demonstrated for over 50 years in large scale applications against agricultural pests. Moreover, it can be easily integrated with other biological control strategies. In terms of the IIT, it has already been proven successful in pilot field trials for suppressing Aedes mosquito vectors.

The pilot field trial of the combined SIT/IIT technology that was reported in this chapter represents the first clear proof-of-concept for the release of sterile male $A e$. aegypti mosquitoes in Thailand (Kittayapong et al. 2019), one of the highly arboviral endemic countries in Southeast Asia. Our results show that the combined SIT/IIT approach for controlling mosquito vectors has potential for practical application as part of integrated vector management, working together with traditional control efforts to achieve better and more efficient outcomes (Zheng et al. 2019).

Potential large-scale application of this integrated SIT/IIT approach is possible through a commitment by the relevant vector control organizations, who should be informed of the technology, especially now that it has already been proven to work well in a pilot field trial.

\section{REFERENCES}

Ageep, T. B., D. Damiens, B. Alsharif, A. Ahmed, E. H. Salih, F. T. Ahmed, A. Diabaté, R. S. Lees, J. R. L. Gilles, and B. B. El Sayed. 2014. Participation of irradiated Anopheles arabiensis males in swarms following field release in Sudan. Malaria Journal 13: 484.

Alphey, L., M. Benedict, R. Bellini, G. G. Clark, D. A. Dame, M. W. Service, and S. L. Dobson. 2010. Sterile-insect methods for control of mosquito-borne diseases: An analysis. Vector Borne and Zoonotic Diseases 10(3): 295-311.

Altinli, M., F. Gunay, B. Alten, M. Weill, and M. Sicard. 2018. Wolbachia diversity and cytoplasmic incompatibility patterns in Culex pipiens populations in Turkey. Parasites \& Vectors 11: 198.

Atyame, C. M., P. Labbé, E. Dumas, P. Milesi, S. Charlat, P. Fort, and M. Weill. 2014. Wolbachia divergence and the evolution of cytoplasmic incompatibility in Culex pipiens. PLoS One 9(1): e87336.

Bian, G., D. Joshi, Y. Dong, P. Lu, G. Zhou, X. Pan, Y. Xu, G. Dimopoulos, and Z. Xi. 2013. Wolbachia invades Anopheles stephensi populations and induces refractoriness to Plasmodium infection. Science 340: 748-751.

Barnes, B. N., J. H. Hofmeyr, S. Groenewald, D. E. Conlong, and M. Wohlfarter. 2015. The Sterile Insect Technique in agricultural crops in South Africa: A metamorphosis .... but will it fly? African Entomology 23(1): 1-18.

Benedict, M. Q., and A. S. Robinson. 2003. The first releases of transgenic mosquitoes: An argument for the sterile insect technique. Trends in Parasitology 19(8): 349-355.

Benedict, M. Q., B. G. J. Knols, H. C. Bossin, P. I. Howell, E. Mialhe, C. Caceres, and A. S. Robinson. 2009. Colonisation and mass rearing: Learning from others. Malaria Journal 8 (Supplement 2): S4. 
Bellini, R., M. Calvitti, A. Medici, M. Carrieri, G. Celli, and S. Maini. 2007. Use of the Sterile Insect Technique against Aedes albopictus in Italy: First results of a pilot trial, pp. 505-515. In M. J. B. Vreysen, A. S. Robinson, and J. Hendrichs (eds.), Area-wide control of insect pests: From research to field implementation. Springer, Dordrecht, The Netherlands.

Bellini, R., A. Medici, A. Puggioli, F. Balestrino, and M. Carrieri. 2013. Pilot field trials with Aedes albopictus irradiated sterile males in Italian urban areas. Journal of Medical Entomology 50(2): $317-$ 325.

Bourtzis, K., S. L. Dobson, Z. Xi, J. L. Rasgon, M. Calvitti, L. A. Moreira, H. C. Bossin, R. Moretti, L. A. Baton, G. L. Hughes, P. Mavingui, and J. R. L. Gilles. 2014. Harnessing mosquito-Wolbachia symbiosis for vector and disease control. Acta Tropica 132: S150-S163.

Brelsfoard, C. L., and S. L. Dobson. 2011. Wolbachia effects on host fitness and the influence of male aging on cytoplasmic incompatibility in Aedes polynesiensis (Diptera: Culicidae). Journal of Medical Entomology 48(5): 1008-1015.

Calvez, E., L. Guillaumot, D. Girault, V. Richard, O. O’Connor, T. Paoaafaite, M. Teurlai, N. Pocquet, V. M. Cao-Lormeau, and M. Dupont-Rouzeyrol. 2017. Dengue-1 virus and vector competence of Aedes aegypti (Diptera: Culicidae) populations from New Caledonia. Parasites \& Vectors 10(1): 381 .

Carvalho, D. O., D. Nimmo, N. Naish, A. R. McKemey, P. Gray, A. B. B. Wilke, M. T. Marrelli, J. F. Virginio, L. Alphey, and M. L. Capurro. 2014. Mass production of genetically modified Aedes aegypti for field releases in Brazil. Journal of Visualized Experiments 83: e3579.

Carvalho, D. O., A. R. McKemey, L. Garziera, R. Lacroix, C. A. Donnelly, L. Alphey, A. Malavasi, and M. L. Capurro. 2015. Suppression of a field population of Aedes aegypti in Brazil by sustained release of transgenic male mosquitoes. PLoS Neglected Tropical Diseases 9(7): e0003864.

Chansang, C., and P. Kittayapong. 2007. Application of mosquito sampling count and geospatial methods to improve dengue vector surveillance. American Journal of Tropical Medicine and Hygiene 77: 897-902.

Clark, M. E., Z. Veneti, K. Bourtzis, and T. L. Karr. 2002. The distribution and proliferation of the intracellular bacteria Wolbachia during spermatogenesis in Drosophila. Mechanisms of Development 111(1): 3-15.

Dame, D. A., D. B. Woodard, H. R. Ford, and D. E. Weidhaas. 1964. Field behavior of sexually sterile Anopheles quadrimaculatus males. Mosquito News 24: 6-14.

Dame, D. A., C. F. Curtis, M. Q. Benedict, A. S. Robinson, and B. G. Knols. 2009. Historical applications of induced sterilisation in field populations of mosquitoes. Malaria Journal 8 (Supplement 2): S2.

Dyck, V. A., J. Hendrichs, and A. S. Robinson (eds.). 2021. Sterile Insect Technique - Principles and practice in Area-Wide Integrated Pest Management. Second Edition. CRC Press, Boca Raton, Florida, USA. $1200 \mathrm{pp}$.

Fredericks, A. C., and A. Fernandez-Sesma. 2014. The burden of dengue and chikungunya worldwide: Implications for the southern United States and California. Annals of Global Health 80(6): 466-475.

Frentiu, F. D., T. Zakir, T. Walker, J. Popovici, A. T. Pyke, A. van den Hurk, E. A. McGraw, and S. L. O'Neill. 2014. Limited dengue virus replication in field-collected Aedes aegypti mosquitoes infected with Wolbachia. PLoS Neglected Tropical Diseases 8(2): e2688.

Gilles, J. R. L., M. F. Schetelig, F. Scolari, F. Marec, M. L. Capurro, G. Franz, and K. Bourtzis. 2014. Towards mosquito sterile insect technique programmes: Exploring genetic, molecular, mechanical and behavioural methods of sex separation in mosquitoes. Acta Tropica 132: S178-S187.

Hilgenboecker, K., P. Hammerstein, P. Schlattmann, A. Telschow, and J. H. Werren. 2008. How many species are infected with Wolbachia? A statistical analysis of current data. FEMS Microbiology Letters 281(2): 215-220.

Hoffmann, A. A., B. L. Montgomery, J. Popovici, I. Iturbe-Ormaetxe, P. H. Johnson, F. Muzzi, M. Greenfield, M. Durkan, Y. S. Leong, Y. Dong, H. Cook, J. Axford, A. G. Callahan, N. Kenny, C. Omodei, E. A. McGraw, P. A. Ryan, S. A. Ritchie, M. Turelli, and S. L. O'Neill. 2011. Successful establishment of Wolbachia in Aedes populations to suppress dengue transmission. Nature 476(7361): 454- 457.

Iyaloo, D. P., K. B. Elahee, A. Bheecarry, and R. S. Lees. 2014. Guidelines to site selection for population surveillance and mosquito control trials: A case study from Mauritius. Acta Tropica 132: S140-S149. 
Joubert, D. A., T. Walker, L. B. Carrington, J. T. De Bruyne, D. H. T. Kien, N. L. T. Hoang, N. V. V. Chau, I. Iturbe-Ormaetxe, C. P. Simmons, and S. L. O'Neill. 2016. Establishment of a Wolbachia superinfection in Aedes aegypti mosquitoes as a potential approach for future resistance management. PLoS Pathogens 12(2): e1005434.

Kittayapong, P., S. Yoksan, U. Chansang, C. Chansang, and A. Bhumiratana. 2008. Suppression of dengue transmission by application of integrated vector control strategies at sero-positive GIS-based foci. American Journal of Tropical Medicine and Hygiene 78: 70-76.

Kittayapong, P., N. Kaeothaisong, S. Ninphanomchai, and W. Limohpasmanee. 2018. Combined sterile insect technique and incompatible insect technique: Sex separation and quality of sterile Aedes aegypti male mosquitoes released in a pilot population suppression trial in Thailand. Parasites \& Vectors 11 (Supplement 2): 657.

Kittayapong, P., S. Ninphanomchai, W. Limohpasmanee, C. Chansang, U. Chansang, and P. Mongkalangoon. 2019. Combined sterile insect technique and incompatible insect technique: The first proof-of-concept to suppress Aedes aegypti vector populations in semi-rural settings in Thailand. PLoS Neglected Tropical Diseases 13(10): e0007771.

Kotsakiozi, P., A. Gloria-Soria, A. Caccone, B. Evans, R. Schama, A. J. Martins, and J. R. Powell. 2017. Tracking the return of Aedes aegypti to Brazil, the major vector of the dengue, chikungunya and Zika viruses. PLoS Neglected Tropical Diseases 11(7): e0005653.

Lees, R. S., D. O. Carvalho, and J. Bouyer. 2021. Potential impact of integrating the Sterile Insect Technique into the fight against disease-transmitting mosquitoes, pp. 1081-1118. In V. A. Dyck, J. Hendrichs, and A. S. Robinson (eds.), Sterile Insect Technique - Principles and practice in Area-Wide Integrated Pest Management. Second Edition. CRC Press, Boca Raton, Florida, USA.

Lees, R. S., J. R. L. Gilles, J. Hendrichs, M. J. B. Vreysen, and K. Bourtzis, K. 2015. Back to the future: The sterile insect technique against mosquito disease vectors. Current Opinion in Insect Science 10: $156-162$.

Mains, J. W., C. L. Brelsfoard, R. I. Rose, and S. L. Dobson. 2016. Female adult Aedes albopictus suppression by Wolbachia-infected male mosquitoes. Scientific Reports 6: 33846.

Mains, J. W., P. H. Kelly, K. L. Dobson, W. D. Petrie, and S. L. Dobson. 2019. Localized control of Aedes aegypti (Diptera: Culicidae) in Miami, FL, via inundative releases of Wolbachia-infected male mosquitoes. Journal of Medical Entomology 56(5): 1296-1303.

Murray, J. V., C. C. Jansen, and P. De Barro. 2016. Risk associated with the release of Wolbachiainfected Aedes aegypti mosquitoes into the environment in an effort to control dengue. Frontiers in Public Health 4: 43.

Moreira, L. A., I. Iturbe-Ormaetxe, J. A. Jeffery, G. J. Lu, A. T. Pyke, L. M. Hedges, B. C. Rocha, S. Hall-Mendelin, A. Day, M. Riegler, L. E. Hugo, K. N. Johnson, B. H. Kay, E. A. McGraw, A. F. van den Hurk, P. A. Ryan, and S. L. O'Neill. 2009. A Wolbachia symbiont in Aedes aegypti limits infection with dengue, chikungunya, and Plasmodium. Cell 139(7): 1268-1278.

Morlan, H. B., E. M. McCray, and J. W. Kilpatrick. 1962. Field tests with sexually sterile males for control of Aedes aegypti. Mosquito News 22: 295-300.

Morrison, N. I., G. Franz, M. Koukidou, T. A. Miller, G. Saccone, and L. S. Alphey. 2010. Genetic improvements to the Sterile Insect Technique for agricultural pests. Asia-Pacific Journal of Molecular Biology and Biotechnology 18(2): 275-295.

(NEA) National Environmental Agency, Singapore. 2016. Risk assessment for the use of male Wolbachia-carrying Aedes aegypti for suppression of the Aedes aegypti mosquito population. Risk Analysis Report of the National Environmental Agency, Singapore, 14 pp.

(NEA) National Environment Agency, Singapore. 2019. New NEA facility to boost production of male Wolbachia-Aedes aegypti mosquitoes to benefit more residents. 2 December 2019.

Nikolouli, K., H. Colinet, D. Renault, T. Enriquez, L. Mouton, P. Gibert, F. Sassu, C. Cáceres, C. Stauffer, R. Pereira, and K. Bourtzis. 2018. Sterile insect technique and symbiosis as potential tools for the control of the invasive Drosophila suzukii. Journal of Pest Science 91(2): 489-503.

O’Connor, L., C. Plichart, A. C. Sang, C. L. Brelsfoard, H. C. Bossin, and S. L. Dobson. 2012. Open release of male mosquitoes infected with a Wolbachia biopesticide: Field performance and infection containment. PLoS Neglected Tropical Diseases 6(11): e1797.

Oliva, C. F., M. Jacquet, J. R. L. Gilles, G. Lemperiere, P. O. Maquart, S. Quilici, F. Schooneman, M. J. B. Vreysen, and S. Boyer. 2012. The sterile insect technique for controlling populations of Aedes albopictus (Diptera: Culicidae) on Réunion Island: Mating vigour of sterilized males. PLoS One 7(11): e49414. 
Papathanos, P. A., H. C. Bossin, M. Q. Benedict, F. Catteruccia, C. A. Malcolm, L. Alphey, and A. Crisanti. 2009. Sex separation strategies: Past experience and new approaches. Malaria Journal 8(2): S2-S5.

Papathanos, P. A., K. Bourtzis, F. Tripet, H. C. Bossin, J. F. Virginio, M. L. Capurro, M. C. Pedrosa, A. Guindo, L. Sylla, M. B. Coulibaly, F. A. Yao, P. S. Epopa, and A. Diabate. 2018. A perspective on the need and current status of efficient sex separation methods for mosquito genetic control. Parasites \& Vectors. 11 (Supplement 2): 654.

Patterson, R. S., V. P. Sharma, K. R. P. Singh, G. C. LaBrecque, P. L. Seetharam, and K. K. Grover. 1975. Use of radio-sterilized males to control indigenous populations of Culex pipiens quinquefasciatus Say: Laboratory and field studies. Mosquito News 35: 1-7.

Patterson, R. S., R. E. Lowe, B. J. Smittle, D. A. Dame, M. D. Boston, and A. L. Cameron. 1977. Release of radiosterilized males to control Culex pipiens quinquefasciatus (Diptera: Culicidae). Journal of Medical Entomology 14: 299-304.

Popovici, J., L. A. Moreira, A. Poinsignon, I. Iturbe-Ormaetxe, D. McNaughton, and S. L. O’Neill. 2010. Assessing key safety concerns of a Wolbachia-based strategy to control dengue transmission by Aedes mosquitoes. Memórias do Instituto Oswaldo Cruz 105(8): 957-964.

Reisen, W. K., M. M. Milby, S. M. Asman, M. E. Bock, R. P. Meyer, P. T. McDonald, and W. C. Reeves. 1982. Attempted suppression of a semi-isolated Culex tarsalis population by the release of irradiated males: A second experiment using males from a recently colonized strain. Mosquito News 42: 565-575.

Ruang-areerate, T., and P. Kittayapong. 2006. Wolbachia transinfection in Aedes aegypti: A potential gene driver of dengue vectors. Proceedings of the National Academy of Science of the USA 103: $12534-12539$.

Sharma, V. P., R. S. Patterson, and H. R. Ford. 1972. A device for the rapid separation of male and female mosquito pupae. Bulletin WHO 47(3): 429-43.

Shepard, D. S., E. A. Undurraga, and Y. A. Halasa. 2013. Economic and disease burden of dengue in Southeast Asia. PLoS Neglected Tropical Diseases 7(2): e2055.

Thomas, D. D., C. A. Donnelly, R. J. Wood, and L. S. Alphey. 2000. Insect population control using a dominant, repressible, lethal genetic system. Science 287(5462): 2474-2476.

Trewin, B. J., J. M. Darbro, C. C. Jansen, N. A. Schellhorn, M. P. Zalucki, T. P. Hurst, and G. J. Devine. 2017. The elimination of the dengue vector, Aedes aegypti, from Brisbane, Australia: The role of surveillance, larval habitat removal and policy. PLoS Neglected Tropical Diseases 11(8): e0005848.

Turelli, M., and A. A. Hoffmann. 1995. Cytoplasmic incompatibility in Drosophila simulans: Dynamics and parameter estimates from natural populations. Genetics 140(4): 1319-1338.

Vreysen M. J. B., J. Gerado-Abaya, and J. P. Cayol. 2007. Lessons from area-wide integrated pest management (AW-IPM) programmes with an SIT component: An FAO/IAEA perspective, pp. 723 744. In M. J. B. Vreysen, A. S. Robinson, and J. Hendrichs (eds.), Area-wide control of insect pests: From research to field implementation. Springer, Dordrecht, The Netherlands.

Vreysen, M. J. B., K. M. Saleh, M. Y. Ali, A. M. Abdulla, Z. R. Zhu, K. G. Juma, V. A. Dyck, A. R. Msangi, P. A. Mkonyi, and H. U. Feldmann. 2000. Glossina austeni (Diptera: Glossinidae) eradicated on the island of Unguja, Zanzibar, using the sterile insect technique. Journal of Economic Entomology 93(1): 123-135.

Weidhaas, D. E., C. H. Schmidt, and E. L. Seabrook. 1962. Field studies on the release of sterile males for the control of Anopheles quadrimaculatus. Mosquito News 22: 283-291.

(WHO) World Health Organization. 2017. Fifth Meeting of the Vector Control Advisory Group, Geneva, Switzerland, 2-4 November 2016. WHO/HTM/NTD/VEM/2017.02. Licence: CC BY-NC-SA 3.0 IGO. Geneva, Switzerland.

(WHO) World Health Organization. 2019a. Dengue and severe dengue. Geneva, Switzerland.

(WHO) World Health Organization. 2019b. Mosquito control: Can it stop Zika at source? Geneva, Switzerland.

Xi, Z., C. C. Khoo, and S. L. Dobson. 2005. Wolbachia establishment and invasion in an Aedes aegypti laboratory population. Science 310(5746): 326-328.

Yakob, L., S. Funk, A. Camacho, O. Brady, and W. J. Edmunds. 2017. Aedes aegypti control through modernized, integrated vector management. PLoS Currents Outbreaks 9: Jan 30.

Zhang, D., Zheng, X., Xi, Z., Bourtzis, K., and J. R. L. Gilles. 2015a. Combining the sterile insect technique with the incompatible insect technique: I. Impact of Wolbachia infection on the fitness of triple and double-infected strains of Aedes albopictus. PLoS One 10: e0121126. 
Zhang, D., R. S. Lees, Z. Xi, J. R. L. Gilles, and K. Bourtzis. 2015b. Combining the sterile insect technique with Wolbachia-based approaches: II. A safer approach to Aedes albopictus population suppression programmes, designed to minimize the consequences of inadvertent female release. PLoS One 10: e0135194.

Zhang, D., R. S. Lees, Z. Xi, K. Bourtzis, and J. R. L. Gilles. 2016. Combining the sterile insect technique with the incompatible insect technique: III. Robust mating competitiveness of irradiated triple Wolbachia-infected Aedes albopictus males under semi-field conditions. PLoS One 11(3): e0151864.

Zhang, D., M. Zhang, Y. Wu, J. R. L. Gilles, H. Yamada, Z. Wu, Z. Xi, and X. Zheng. 2017. Establishment of a medium-scale mosquito facility: Optimization of the larval mass-rearing unit for Aedes albopictus (Diptera: Culicidae). Parasites \& Vectors 10: 569.

Zheng, M. L., D. J. Zhang, D. D. Damiens, R. S. Lees, and J. R. L. Gilles. 2015. Standard operating procedures for standardized mass rearing of the dengue and chikungunya vectors Aedes aegypti and Aedes albopictus (Diptera: Culicidae): II. Egg storage and hatching. Parasites \& Vectors 8: 348.

Zheng, X., D. Zhang, Y. Li, Y. Wu, X. Liang, Y. Liang, X. Pan, L. Hu, Q. Sun, X. Wang, Y. Wei, J. Zhu, W. Qian, Z. Yan, A. G. Parker, J. R. L. Gilles, K. Bourtzis, J. Bouyer, M. Tang, B. Zheng, J. Yu, J. Liu, J. Zhuang, Z. Hu, M. Zhang, J. Gong, X. Hong, Z. Zhang, L. Lin, Q. Liu, Z. Hu, Z. Wu, L. A. Baton, A. A. Hoffmann, and Z. Xi. 2019. Incompatible and sterile insect techniques combined eliminate mosquitoes. Nature 572: 56-61. 